Querverbindungen die zusätzliche Stromwärme wesentlich verringern können. In allen Fällen läßt sie sich durch geeignete Verschränkung innerhalb der Nut oder durch magnetische Hilfskreise vollkommen unterdrücken. Die Leistung der Maschinen und die Ausnützung der Baustoffe werden nicht mehr durch die zusätzliche Stromwärme begrenzt, denn bei zweckmäßig entworfenen Wicklungen mit unterteilten Leitern gibt es keine kritische Leiterhöhe, bei deren Überschreitung die Stromwärme wieder zunimmt.

\title{
Die Bestimmung kleiner Phasenverschiebungen bei Niederfrequenz.
}

\author{
Von \\ C. Déguisne, Frankfurt a. M.
}

Die Messung von Phasenverschiebungen hat bisher, sobald es sich um kleinere Werte handelte, an die Geschicklichkeit des Beobachters besondere Anforderungen gestellt. Meist mußte, um genügende Empfindlichkeit der Meßanordnung zu erreichen, Wechselstrom höherer Frequenz benutzt werden. Da die Erzeugung dieses Stromes in genügend reiner Sinusform Schwierigkeiten bereitet, gaben die Oberschwingungen oft zu Störungen und Fehlern Anlaß. Die hohe Empfindlichkeit des ScheringSchmidtschen Vibrationsgalvanometers ${ }^{1}$ ) ermöglicht nunmehr, solche Messungen mit Wechselstrom von der in der Technik üblichen Frequenz mit ausreichender Genauigkeit durchzuführen. Die Spannungen, die die heutigen Wechselstromzentralen uns zur Verfügung stellen, haben - abgesehen von den hochfrequenten Nutenharmonischen geringer Amplitude, die sich durch Vorschalten einer Drosselspule leicht unschädlich machen lassen - meist eine der Sinusform so nahe kommende Kurve, daß sie zu den genannten Messungen ohne weiteres verwendet werden können. Die Benutzung der Netzspannung hat nebenher den Vorzug großer Konstanz der Frequenz, was gerade bei Verwendung des Vibrationsgalvanometers von Wert ist.

Einige Meßanordnungen, welche die Bestimmung von Phasenverschiebungen bei Niederfrequenz bezwecken, sind vor kurzem vom Verfasser ${ }^{2}$ ) behandelt worden. Es wurde dabei an Stelle eines regulierbaren Kondensators die Verwendung einer veränderlichen gegenseitigen Induktivität empfohlen; sie ist dort unter der Bezeichnung „Phasenschlitten“ im Absatz I, 2 beschrieben. Es soll nun im Folgenden dargelegt werden, wie die Benutzung des Phasenschlittens auch die Messung in der Schaltung der Thomson-Brücke recht einfach gestaltet.

Der zu untersuchende Widerstand AC sei, wie. in Abb. I dargestellt, mit dem Vergleichswiderstand $\mathrm{A}^{\prime} \mathrm{C}^{\prime}$ in Reihe geschaltet. Die Widerstände der Thomsonschen Brückenanordnung seien $r, r^{\prime}, w$ und $w^{\prime}$. Sie seien induktions- und kapazitätsfrei. Zwischen den Punkten $S$ und $T$ liege das Vibrationsgalvanometer. Für die Strombahn

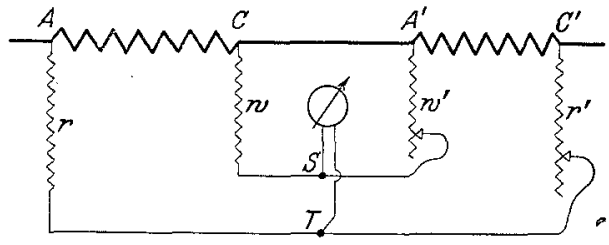

Abb. I. AC soll der Werkwiderstand $\mathrm{R}$ und die Phasenverschiebung $\alpha$ zwischen Strom und Klemmenspannung bestimmt, bzw. auf die entsprechenden Werte $\mathrm{R}^{\prime}$ und $a^{\prime}$ des Vergleichswiderstands $\mathrm{A}^{\prime} \mathrm{C}^{\prime}$ zurückgeführt werden, wobei auch der Fall $\alpha^{\prime}=0$ nicht ausgeschlossen sei.

1) Schering und Schmidt, diese Zeitschr. 1, S. 254, I9I2.

$\left.{ }^{2}\right)$ Déguisne, diese Zeitschr. 5, S. 303, 1917. 
Wir schicken durch die Strombahn $\mathrm{ACA}^{\prime} \mathrm{C}^{\prime}$ einen sinusförmigen Wechselstrom J mit der Kreisfrequenz $\omega$. Die dann auftretenden Spannungen sind durch das Vektorendiagramm der Abb. 2 nach Größe und Phase dargestellt. Die Richtung AB entspreche der Phase des Stromes J. Dann stellt der Vektor AB die Werkspannung J.R dar.

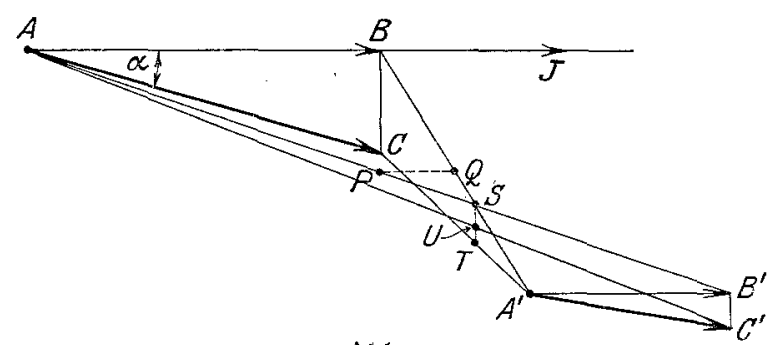

Abb. 2 . $A C$ ist die, an den Klemmen der Strombahn AC in Abb. I auftretende Spannung und $\Varangle \mathrm{BAC}=\imath$. Analog sind die Vektoren $A^{\prime} B^{\prime}=J \cdot R^{\prime}$ und $\Varangle \mathrm{B}^{\prime} \mathrm{A}^{\prime} \mathrm{C}^{\prime}=a^{\prime}$. Die Vektoren $\mathrm{AC}^{\prime}$ und $\mathrm{CA}^{\prime}$ geben die Spannungen wieder, die über die Widerstände $r$ und $r^{\prime}$ bzw. $w$ und $w^{\prime}$ der Abb. I auftreten. Für die Verbindungsleitung $\mathrm{CA}^{\prime}$ ist hier der Über-

sichtlichkeit halber eine größere Induktivität angenommen, so daß der ihr entsprechende Vektor $\mathrm{CA}^{\prime}$ mit der Richtung des Stromvektors AB einen größeren Winkel bildet, als dies bei den Vektoren $A C$ und $A^{\prime} C^{\prime}$ der Fall ist. Die Größe dieses Winkels kommt für die nachstehende Ableitung zunächst nicht in Betracht; er kann ebenso gut $=0$ oder negativ angesetzt werden.

Zur Einfuhrung in unsere Betrachtungsweise nehmen wir zunächst an, es sei $\alpha=0$ und auch $a^{\prime}=0$. Dann treten an Stelle von $\mathrm{AC}^{\prime}$ und $\mathrm{CA}^{\prime}$ die Vektoren $\mathrm{AB}^{\prime}$ und BA'. Wir nehmen ferner an, daß wir entsprechend der für die Thomsonbrücke geltenden Vorschrift die Brückenwiderstände stets so regulieren, daß

$$
\mathrm{r}: \mathrm{r}^{\prime}=\mathrm{w}: \mathrm{w}^{\prime}
$$

bleibt. Das Potential des Punktes $T$ der Abb. I werde in Abb. 2 durch den Punkt $P$ und das von $\mathrm{S}$ durch $\mathrm{Q}$ dargestellt. $\mathrm{PQ}$ gibt dann die Potentialdifferenz wieder, die auf das Galvanometer wirkt. Da entsprechend der oben gemachten Vorschrift über die Regulierung der Brückenwiderstände stets

$$
\frac{\mathrm{AP}}{\mathrm{PB}^{\prime}}=\frac{\mathrm{BQ}}{\mathrm{QA}^{\prime}}
$$

bleibt, so ist $\mathrm{PQ}$ stets parallel mit $\mathrm{AB}$, und wenn bei der Regulierung $\mathrm{P}$ bis nach dem Schnittpunkt $\mathrm{S}$ gewandert ist, so ist auch $\mathrm{Q}$ daselbst angekommen, d. h. PQ ist gleich Null geworden und das Galvanometer ist stromlos. Dann ist auch

$$
\mathrm{AB}: \mathrm{A}^{\prime} \mathrm{B}^{\prime}=\mathrm{AS}: \mathrm{SB}^{\prime}=\mathrm{r}: \mathrm{r}^{\prime} \text {. }
$$

Es werde nun die Einschränkung $\ell=0$ fallen gelassen; dagegen sei wie oben $\alpha^{\prime}=0$. Nun tritt an Stelle von AB der Vektor AC und an Stelle von BA' der Vektor $\mathrm{CA}^{\prime}$. Da aber $\mathrm{C}$ senkrecht unter $\mathrm{B}$ liegt, so haben sich sämtliche Punkte von $\mathrm{BA}^{\prime}$ in zu AB senkrechter Richtung nach unten verschoben. Wenn nunmehr durch die Regulierung der Punkt $P$ nach $S$ gelangt ist, befindet sich $Q$ nicht in $S$, sondern in $\mathrm{T}$, wobei ST senkrecht zu AB steht; d. h. wenn das Verhältnis der Brïckenwiderstände gleich $\mathrm{AB}: \mathrm{A}^{\prime} \mathrm{B}^{\prime}$ gemacht worden ist, wird das Galvanometer nicht stromlos, sondern es steht unter einer Spannung, die dem Strom J um $90^{\circ}$ voreilt. Diese Spannung ST kann aber mit Hilfe des Phasenschlittens kompensiert werden.

Der Phasenschlitten ${ }^{1}$ ) besteht aus zwei ineinander verschiebbaren Spulensätzen $S_{1}$ und $S_{2}$. Fließt in $S_{1}$ ein Wechselstrom, so entsteht in $S_{2}$ eine EMK, die zum Strome senkrecht steht und deren Größe dem Strom proportional ist, andererseits aber von der gegenseitigen Einstellung der beiden Spulen abhängt. Schalten wir die Spule $\mathrm{S}_{\mathbf{1}}$

1) Herstellung und Vertrieb des Phasenschlittens ist von der Hartmann \& Braun A.-G., Frankfurt a. M. tibernommen worden. 
in Reihe mit $\mathrm{AC}$, also etwa in die Verbindungsleitung $\mathrm{CA}^{\prime}$, so wird sie, wenn wir den uber $w$ und $w^{\prime}$ abfließenden Strom zunächst als verschwindend klein gegenüber dem. Strom J voraussetzen, von einem Strom von der Phase $A B$ durchflossen, und legen wir die Spule $\mathrm{S}_{2}$ in den Galvanometerkreis - die Schaltung ist in Abb. 3 wiedergegeben - so läßt sich durch gegenseitige Verschiebung der Spulen die Spannung ST kompensieren und der Ausschlag des Galvanometers auf Null bringen.

Bevor die zur Berechnung von $a$ führenden Gleichungen abgeleitet werden, soll

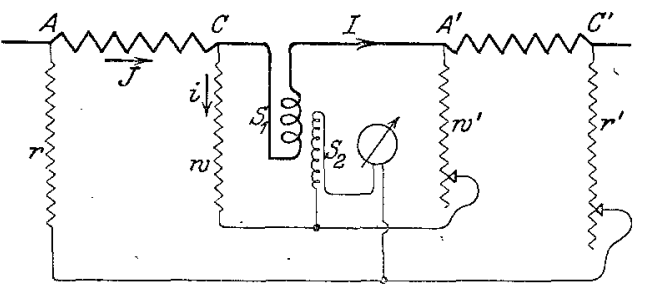

Abb. 3 . noch die zweite Einschränkung $a^{\prime}=0$ beseitigt werden. Besitzt der Normalwiderstand $\mathrm{A}^{\prime} \mathrm{C}^{\prime}$ ebenfalls Induktivität oder Kapazität, so wird in $\mathrm{Abb} .2$ seine Klemmenspannung durch den Vektor $\mathrm{A}^{\prime} \mathrm{C}^{\prime}$ dargestellt, und an Stelle von $\mathrm{AB}^{\prime}$ tritt der Vektor $\mathrm{A} \mathrm{C}^{\prime}$, wobei wiederum $\mathrm{B}^{\prime} \mathrm{C}^{\prime}$ senkrecht zu $\mathrm{AB}$ steht. Damit rücken sämtliche Punkte des Vektors $A C^{\prime}$ senkrecht nach unten. Insbesondere fällt der Punkt, in $\operatorname{dem} \mathrm{AC}^{\prime} \mathrm{im}$ Verhältnis $r: r^{\prime}$ geteilt wird, statt nach $S$ nach $U$. Die auf das Galvanometer wirkende Spannung ist nunmehr UT. Auch sie ist gegen AB um $90^{\circ}$ verschoben und kann durch den Phasenschlitten kompensiert werden.

Nach dem bisher Gesagten hat der Gang der Messung in folgender Weise zu geschehen: Zugrunde gelegt ist die Schaltung der Abb. 3. Die Brückenwiderstände werden bei ausgezogenem Phasenschlitten derart reguliert, daß stets $r: r^{\prime}=w^{\prime}: w^{\prime}$ ist, so lange, bis der Galvanometerausschlag ein Minimum erreicht. Hierauf wird der Phasenschlitten eingeschoben und die Spule $S_{2}$ in dem Sinne geschaltet, daß dabei der Ausschlag des Galvanometers kleiner wird. Die Einstellung des Schlittens, bei welcher der Ausschlag verschwindet, wobei die Brückenwiderstände, wenn erforderlich, etwas nachzuregulieren sind, heiße $\eta$.

Aus Abb. 2 ergeben sich nun folgende Beziehungen:

oder da

$$
\mathrm{AB}: \mathrm{A}^{\prime} \mathrm{B}^{\prime}=\mathrm{AS}: \mathrm{SB}^{\prime}=\mathrm{AU}: \mathrm{UC}^{\prime}
$$

ist

$$
\mathrm{AB}=\mathrm{R} \cdot \mathrm{J}, \quad \mathrm{A}^{\prime} \mathrm{B}^{\prime}=\mathrm{R}^{\prime} \cdot \mathrm{J} \text { und } \mathrm{AU}: \mathrm{UC}^{\prime}=\mathrm{r}: \mathrm{r}^{\prime} \text {, }
$$

Ferner ist

$$
\mathrm{R}: \mathrm{R}^{\prime}=\mathrm{r}: \mathrm{r}^{\prime} \text {. . . . . . . . . . . . . . I I) }
$$

$$
\operatorname{tg} u=\frac{\mathrm{BC}}{\mathrm{AB}}
$$

Es ist aber

folglich :

$$
\mathrm{BC}: \mathrm{ST}=\mathrm{CA}^{\prime}: \mathrm{TA}^{\prime}=\left(\mathrm{r}+\mathrm{r}^{\prime}\right) / \mathrm{r}^{\prime}
$$

Nun ist aber

$$
\mathrm{BC}=\mathrm{ST} \cdot\left(\mathrm{r}+\mathrm{r}^{\prime}\right) / \mathrm{r}^{\prime}
$$

und wie oben

$$
\mathrm{ST}=\mathrm{UT}+\mathrm{SU}
$$

also ist

$$
\mathrm{SU}=\mathrm{B}^{\prime} \mathrm{C}^{\prime} \cdot \mathrm{r} /\left(\mathrm{r}+\mathrm{r}^{\prime}\right)
$$

$$
\mathrm{BC}=\mathrm{UT} \cdot \frac{\mathrm{r}+\mathrm{r}^{\prime}}{\mathrm{r}^{\prime}}+\mathrm{B}^{\prime} \mathrm{C}^{\prime} \cdot \frac{\mathrm{r}}{\mathrm{r}^{\prime}} \text {. }
$$

Andererseits ist nach Gl. I) und den vorangehenden Gleichungen

$$
A B=A^{\prime} B^{\prime} \cdot \frac{r}{r^{\prime}}
$$


Setzen wir jetzt in die Gleichung für $\operatorname{tg} \alpha$ die für $\mathrm{BC}$ und $\mathrm{AB}$ gefundenen Ausdrücke ein, so wird

$$
\operatorname{tg} \alpha=\frac{\mathrm{U}^{\prime} \mathrm{T}}{\mathrm{A}^{\prime} \mathrm{B}^{\prime}} \cdot \frac{\mathrm{r}+\mathrm{r}^{\prime}}{\mathrm{r}}+\frac{\mathrm{B}^{\prime} \mathrm{C}^{\prime}}{\mathrm{A}^{\prime} \mathrm{B}^{\prime}}
$$

Nun ist aber, wenn $\eta$ die Einstellung ist, welche an der mit der benutzten Frequenz geeichten Skala des Phasenschlittens abgelesen wird,

Ferner ist

$$
\mathrm{UT}=\eta \cdot \mathrm{J}
$$

und

$$
\mathrm{A}^{\prime} \mathrm{B}^{\prime}=\mathrm{R}^{\prime} \cdot \mathrm{J}
$$

Demnach wird

$$
\frac{\mathrm{B}^{\prime} \mathrm{C}^{\prime}}{\mathrm{A}^{\prime} \mathrm{B}^{\prime}}=\operatorname{tg} \alpha^{\prime}
$$

$$
\left.\operatorname{tg} \alpha=\frac{\eta}{\mathrm{R}^{\prime}} \cdot\left(\mathrm{I}+\frac{\mathrm{r}^{\prime}}{\mathrm{r}}\right)+\operatorname{tg} \cdot \alpha^{\prime} . . . . . . .2\right)
$$

$\mathrm{Ob}$ die gemessene Phasenverschiebung induktiv oder kapazitiv ist, ergibt sich aus dem Sinn, in dem die Spulen des Phasenschlittens in den Stromkreis einzuschalten sind, um das Galvanometer auf Null zu bringen. Dieser Sinn wird durch einen Vorversuch ein für alle Mal festgestellt und an den Klemmen des Phasenschlittens angemerkt, etwa in der Weise, daß die Klemmen des Apparats, welche bei vorherrschender Induktivität der Strombahn $\mathrm{AC}$ an die Punkte $\mathrm{C}$ und $\mathrm{T}$ der Abb. I anzuschließen sind, mit dem +Zeichen versehen werden. Müssen dann bei einer Messung die Klemmen der Spule $\mathrm{S}_{2}$ des Phasenschlittens vertauscht werden, so ist in Gl. 2) $\eta$ mit dem - Zeichen einzusetzen, und bei den Werten für $\operatorname{tg} a$ und $\operatorname{tg} \alpha^{\prime}$ ist dem induktiven Verhalten das +-, dem kapazitiven das — Zeichen zuzuordnen.

Die Gleichungen I) und 2) sind unter der Annahme abgeleitet, daß der durch die Spule $S_{1}$ des Phasenschlittens fließende Strom mit dem Strome in $A C$ und $A^{\prime} C^{\prime}$ identisch sei. Dies schlitten in die eine dieser Strombahnen direkt einschalten. Wir würden uns damit der Vorzüge der Thomsonschen Brückenschaltung, die es ermöglicht, die von den Verbindungsleitungen herrührenden, nicht kontrollierbaren Fehler zu vermeiden, zum Teil berauben. Wir ziehen es daher vor, den Phasenschlitten so zu schalten, wie in Abb. 3 vorgeschlagen, nämlich in die Verbindungsleitung $\mathrm{CA}^{\prime}$, und die dadurch bedingten Korrekturen für die Gleichungen I) und 2) festzustellen. Dies soll im folgenden geschehen.

Parallel zur Strombahn CA' liegt der Widerstand $w+w^{\prime}$. Der Strom in AC bzw. $\mathrm{A}^{\prime} \mathrm{C}^{\prime}$ ist die Resultierende aus den durch die beiden parallelen Stromzweige fließenden Strömen. Abb. 4 gibt das Stromdiagramm hierzu. OP sei der in $\mathrm{CA}^{\prime}$, also in der

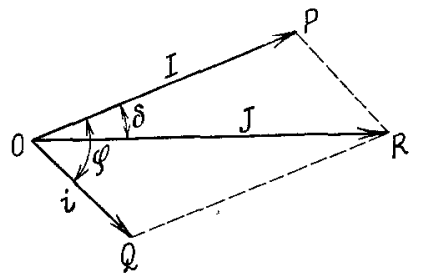

Abb. 4 . Spule $S_{1}$ des Phasenschlittens fließende, Strom; er heiße I. $O Q$ sei der Strom $i$, der von den Widerständen $w$ und $\mathrm{w}^{\prime}$ aufgenommen wird, und ihre Resultierende $\mathrm{OR}$ ist der Strom $J$ in den Bahnen $A C$ und $A^{\prime} C^{\prime}$. Der Strom i ist in Phase mit der Potential-Differenz $\mathrm{CA}^{\prime}$, da die Widerstände $\mathrm{w}$ und $\mathrm{w}^{\prime}$ induktions- und kapazitätsfrei sind. Der Winkel POQ — er heiße $\phi$ — wird daher durch die Eigenschaften des Zweiges CA' bestimmt. Der Widerstand dieses $Z$ weiges sei $W$, seine Induktivität $L$. Seine Kapazität werde zur Vereinfachung der Rechnung gleich Null angenommen; ihr Einfluß würde, von Sonderfällen abgesehen, in unserer Ableitung, wo es sich um Korrektionsgrößen handelt, 
verschwindend klein sein. Zunächst soll die Größe des Winkels POR, mit $\delta$ bezeichnet, gefunden werden.

Im Dreieck OPR ist $\Varangle \mathrm{PRO}=\varphi-\delta$ und daher

$$
\frac{\sin (\varphi-\delta)}{\sin \delta}=\frac{\mathrm{I}}{\mathrm{i}} \text {. }
$$

Wir schreiben nun:

$$
\frac{\sin (\varphi-\delta)}{\sin \delta}=\sin \varphi \cdot \cot \delta-\cos \varphi .
$$

Also

Es ist aber

$$
\cot \delta=\frac{\mathrm{I}}{\mathrm{i}} \cdot \frac{\mathrm{I}}{\sin \varphi}+\cot \varphi .
$$

ferner

$$
\frac{\mathrm{I}}{\mathrm{i}}=\frac{\mathrm{w}+\mathrm{w}^{\prime}}{\sqrt{\mathrm{W}^{2}+(\omega \mathrm{L})^{2}}} \text { und } \frac{\mathrm{I}}{\sin \varphi}=\frac{\sqrt{\mathrm{W}^{2}}+(\omega \mathrm{L})^{2}}{\omega \mathrm{L}}
$$

Also wird

$$
\cot \varphi=\frac{\mathrm{W}}{\omega \mathrm{L}} .
$$

oder

$$
\cot \delta=\frac{\mathrm{w}+\mathrm{w}^{\prime}}{\omega \mathrm{L}}+\frac{\mathrm{W}}{\omega \mathrm{L}}
$$

$$
\left.\operatorname{tg} \delta=\frac{\omega \mathrm{L}}{\mathrm{w}+\mathrm{w}^{\prime}+\mathrm{W}} \cdot . . . . . . . .3\right)
$$

Wir berechnen nunmehr das Verhältnis I:J und setzen zu diesem Zwecke aus Dreieck OPR in Abb. 4 an:

oder

$$
\mathrm{J}^{2}=\mathrm{I}^{2}+\mathrm{i}^{2}+2 \cdot \mathrm{I} \cdot \mathrm{i} \cdot \cos \varphi
$$

$$
\left(\frac{J}{\mathrm{I}}\right)^{2}=\mathrm{I}+\left(\frac{\mathrm{i}}{\mathrm{I}}\right)^{2}+2 \cdot \frac{\mathrm{i}}{\mathrm{I}} \cdot \cos \varphi
$$

Da aber

so wird

$$
\frac{\mathrm{i}}{\mathrm{I}}=\frac{\sqrt{\mathrm{W}^{2}+(\omega \overline{\mathrm{L}})^{2}}}{\mathrm{w}+\mathrm{w}^{\prime}} \text { und } \cos \varphi=\frac{\mathrm{W}}{\sqrt{\mathrm{W}^{2}}+\frac{\mathrm{L})^{2}}{}}
$$

$$
\left(\frac{J}{I}\right)^{2}=I+\frac{W^{2}+(\omega L)^{2}}{\left(w+w^{\prime}\right)^{2}}+2 \cdot \frac{W}{w+w^{\prime}}
$$

und nach einigen Umrechnungen

$$
\frac{J}{I}=\frac{W+w+w^{\prime}}{w+w^{\prime}}\left[I+1 / 2\left(\frac{w L}{w+w^{\prime}+W}\right)^{2}-\frac{I}{8}\left(\frac{w L}{w+w^{\prime}+W}\right)^{4}+. . .\right] \text {. }
$$

Mit Rücksicht darauf, daß das Glied $\frac{\omega L}{w+w^{\prime}+W}$, wie sich weiterhin noch ergeben wird, sehr klein bleibt, können wir in der Klammer des obigen Ausdrucks seine vierte und höheren Potenzen vernachlässigen und können die Gleichung umformen in:

oder auch nach Gl. 3)

$$
\frac{\mathrm{I}}{\mathrm{J}}=\frac{\mathrm{w}+\mathrm{w}^{\prime}}{\mathrm{w}+\mathrm{w}^{\prime}-\mathrm{W}} \cdot\left[\mathrm{I}-1 / 2 \cdot\left(\frac{\mathrm{wL}}{\mathrm{w}+\mathrm{w}^{\prime}+\mathrm{W}}\right)^{2}\right]
$$

$$
\frac{\mathrm{I}}{\mathrm{J}}=\frac{\mathrm{w}+\mathrm{w}^{\prime}}{\mathrm{w}+\mathrm{w}^{\prime}+\mathrm{W}} \cdot\left(\mathrm{I}-1 / 2 \cdot \operatorname{tg}^{2} \delta\right) .
$$

Über den Wert des Klammerausdrucks dieser Gleichung ist folgendes 'zu sagen. Die Widerstände $w$ und $w^{\prime}$ werden dank der hohen Empfindlichkeit des Vibrations- 
galvanometers stets von der Größenordnung Ioo Ohm gewählt werden können, so daß im Ausdruck für tg $\delta$ der Gl. 3) der Nenner größer als I30 bis I50 Ohm ist. L d. i. die Induktivität der Verbindungsleitung $\mathrm{CA}^{\prime}$ in Abb. 3, kann leicht kleiner als 0,002 Henry gehalten werden; die Induktivität des hier eingeschalteten Phasenschlittens beträgt weniger als 0,00 I Henry. Bei.5o Perioden, d. h. $\omega=3$ I 4 , läßt sich also $\operatorname{tg} \delta$ bequem kleiner als 0,005 machen. Der Klammerausdruck hat demnach unter diesen Verhältnissen auf den Wert von $\frac{I}{J}$ keinen merkbaren. Einfluß, und wir können setzen:

$$
\frac{\mathrm{I}}{\mathrm{J}}=\frac{\mathrm{w}+\mathrm{w}^{\prime}}{\mathrm{w}+\mathrm{w}^{\prime}+\mathrm{W}} \text {. }
$$

Diese Beziehung veranlaßt uns, die Gl. 2) folgendermaßen abzuändern. Bei ihrer Ableitung war das Verhältnis

$$
\frac{\mathrm{UT}}{\mathrm{A}^{\prime} \mathrm{B}^{\prime}}=\frac{\eta \cdot \mathrm{J}}{\mathrm{R}^{\prime} \cdot \mathrm{J}}
$$

gesetzt worden. Nach dem obigen tritt als Faktor von $\eta$ der Strom I anstelle von J, und das Verhältnis wird

$$
\frac{\mathrm{U}^{\top} \mathrm{T}}{\mathrm{A}^{\prime} \mathrm{B}^{\prime}}=\frac{\eta}{\mathrm{R}^{\prime}} \cdot \frac{\mathrm{w}+\mathrm{w}^{\prime}}{\mathrm{w}+\mathrm{w}^{\prime}+\mathrm{W}} .
$$

Die Gl. 2) geht also über in:

$$
\operatorname{tg} a=\frac{\eta}{\mathrm{R}^{\prime}} \cdot \frac{\mathrm{w}+\mathrm{w}^{\prime}}{\mathrm{w}+\mathrm{w}^{\prime}+\mathrm{W}} \cdot\left(\mathrm{I}+\frac{\mathrm{r}^{\prime}}{\mathrm{r}}\right)+\operatorname{tg} a^{\prime}
$$

An dieser Gleichung ist noch eine weitere Korrektur wegen der Winkel $a$ und $\alpha^{\prime}$ anzubringen. $\alpha$ sollte den Phasenwinkel zwischen der Klemmenspannung und dem Strom J der Bahn AC bezeichnen. Da nun aber nach dem Diagramm der Abb. 4 die Richtung des Stromes J um den Winkel $\delta$ von dem Strom I abweicht, im Diagramm

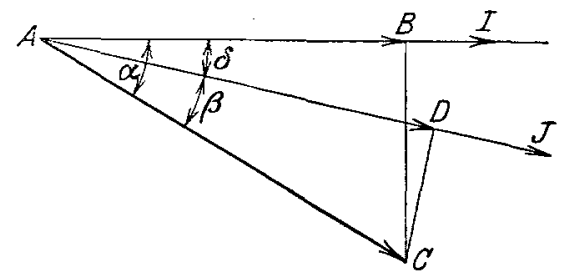

Abb. 5 . der Abb. 2 aber der Vektor AB nunmehr als mit dem Strom I gleichgerichtet anzunehmen ist, so stellt der dortige Winkel BAC nicht die gesuchte Phasenverschiebung zwischen Strom und Klemmenspannung dar; die Abb. 2 (linke Hälfte) geht vielmehr über in die Abb. 5. Hier sei AC die Klemmenspannung, AD deren Komponente in Richtung des Stromes J; sie heiße die Werkspannung und ist gleich $J \cdot R$, wenn $R$ der Werkwiderstand der zu untersuchenden Strombahn ist. Der Vektor AB falle entsprechend der für Abb. 2 gemachten Annahme in die Richtung des den Phasenschlitten durchfließenden Stroms I. Die gesuchte Phasenverschiebung wird dann durch den Winkel DAC dargestellt; er heiße jetzt vorübergehend $\beta$. Es ist also

$$
\alpha=\beta+\delta \text {. }
$$

Die gleichen Beziehungen gelten für die Strombahn $\mathrm{A}^{\prime} \mathrm{C}^{\prime}$, wo die Verschiebung żwischen Strom J und Klemmspannung $\mathrm{A}^{\prime} \mathrm{C}^{\prime}$ nunmehr mit $\beta^{\prime}$ bezeichnet worde. Die Gleichung für $\operatorname{tg} \alpha$ nimmt nun nachstehende. Form an:

$$
\operatorname{tg}(\beta+\delta)=\frac{\eta}{\mathrm{R}^{\prime}} \cdot \frac{\mathrm{w}+\mathrm{w}^{\prime}}{\mathrm{w}+\mathrm{w}^{\prime}+\mathrm{W}} \cdot\left(\mathrm{I}+\frac{\mathrm{r}^{\prime}}{\mathrm{r}}\right)+\operatorname{tg}\left(\beta^{\prime}+\delta\right) .
$$

Wir formen sie um in:

$\operatorname{tg} \beta+\operatorname{tg} \delta=\frac{\eta}{\mathrm{R}^{\prime}} \cdot \frac{\mathrm{w}+\mathrm{w}^{\prime}}{\mathrm{w}+\mathrm{w}^{\prime}+\mathrm{W}}\left(\mathrm{I}+\frac{\mathrm{r}^{\prime}}{\mathrm{r}}\right) \cdot(\mathrm{I}-\operatorname{tg} \beta \cdot \operatorname{tg} \delta)+\left(\operatorname{tg} \beta^{\prime}+\operatorname{tg} \delta\right) \cdot \frac{\mathrm{I}-\operatorname{tg} \beta \cdot \operatorname{tg} \delta}{\mathrm{I}-\operatorname{tg} \beta^{\prime} \cdot \operatorname{tg} \delta} \cdot$ 
Nun ist aber

$$
\frac{\mathrm{I}-\operatorname{tg} \beta \cdot \operatorname{tg} \delta}{\mathrm{I}-\operatorname{tg} \beta^{\prime} \cdot \operatorname{tg} \delta}=\mathrm{I}-\frac{\left(\operatorname{tg} \beta-\operatorname{tg} \beta^{\prime}\right) \cdot \operatorname{tg} \delta}{\mathrm{I}-\operatorname{tg} \beta^{\prime} \operatorname{tg} \delta} .
$$

Wie oben ausgeführt, kann $\operatorname{tg} \delta$ leicht kleiner als 0,005 gehalten werden. Liegt $\operatorname{tg} \beta-\operatorname{tg} \beta^{\prime}$ unter dem Werte 0,2 , so dürfen wir den obigen Ausdruck mit I gleichsetzen, ohne daß der Fehler für $\operatorname{tg} \beta \mathrm{I} \%$ übersteigt. Nur wenn $\operatorname{tg} \beta$ wesentlich kleiner als $\operatorname{tg} \beta^{\prime}$ wird und sich dem Wert o nähert, kann die Vernachlässigung unzulässig werden. Im ersten Fall nimmt die Gleichung für $\operatorname{tg} \beta$ die Form an:

$$
\left.\operatorname{tg} \beta=\frac{\eta}{\mathrm{R}^{\prime}} \cdot\left(\mathrm{I}+\frac{\mathrm{r}^{\prime}}{\mathrm{r}}\right) \frac{\mathrm{w}+\mathrm{w}^{\prime}}{\mathrm{w}+\mathrm{w}^{\prime}+\mathrm{W}} \cdot(\mathrm{I}-\operatorname{tg} \beta \cdot \operatorname{tg} \delta)+\operatorname{tg} \beta^{\prime} . \quad . \quad . \quad . \quad .4 \mathrm{a}\right)
$$

Auch der Klammerausdruck $(I-\operatorname{tg} \beta \cdot \operatorname{tg} \delta)$ ist mit einer Annäherung von $I_{0}^{0}$ gleich I, wenn $\operatorname{tg} \beta$ selbst unterhalb 0,2 liegt. Die Berechnung von $\operatorname{tg} \beta$ erfolgt in der Weise, daß, der Wert zunächst ohne Berücksichtigung des Klammerausdrucks in erster Annäherung bestimmt, dann die Größe $\operatorname{tg} \beta \cdot \operatorname{tg} \delta$ berechnet und nun mit Rücksicht auf die gewünschte Meßgenauigkeit festgestellt wird, ob die Anbringung der Korrektur erforderlich ist.

Ebenso ist noch die Gültigkeit der Gleichung I) zu untersuchen. Wie aus dem Diagramm in Abb. 2 oben abgeleitet worden ist, verhalten sich nach erfolgter Einstellung der Brücke die Widerstände $r: r^{\prime}$ wie die Vektoren $A B: A^{\prime} B^{\prime}$. Nun sind diese aber nach den bisherigen Ausführungen nicht identisch mit den Werkspannungen. Wollen wir das Verhältnis dieser, bzw. das Verhältnis der Werkwiderstände finden, so müssen wir in folgender Weise umrechnen. In Abb. 5 ist die Werkspannung durch den Vektor AD wiedergegeben. Nun ist aber

$$
\mathrm{AB}: \mathrm{AD}=\cos \alpha: \cos \beta \text {. }
$$

$$
\cos c: \cos \beta=\cos (\beta+\delta): \cos \beta=\cos \delta-\operatorname{tg} \beta \cdot \sin \delta \text {. }
$$

Also ist

Ebenso ist

$$
\mathrm{AB}: \mathrm{AD}=\cos \delta-\operatorname{tg} \beta \cdot \sin \delta .
$$

Daraus folgt

$$
\mathrm{A}^{\prime} \mathrm{B}^{\prime}: \mathrm{A}^{\prime} \mathrm{D}^{\prime}=\cos \delta-\operatorname{tg} \beta^{\prime} \cdot \sin \delta .
$$

$$
\frac{\mathrm{AD}}{\mathrm{A}^{\prime} \mathrm{D}^{\prime}}=\frac{\mathrm{AB}}{\mathrm{A}^{\prime} \mathrm{B}^{\prime}} \cdot \frac{\cos \delta-\operatorname{tg} \beta^{\prime} \cdot \sin \delta}{\cos \delta-\operatorname{tg} \beta \cdot \sin \delta}=\frac{\mathrm{AB}}{\mathrm{A}^{\prime} \mathrm{B}^{\prime}} \cdot \frac{\mathrm{I}-\operatorname{tg} \beta^{\prime} \cdot \operatorname{tg} \delta}{\mathrm{I}-\operatorname{tg} \beta \cdot \operatorname{tg} \delta} .
$$

Und da

$$
\frac{A D}{A^{\prime} D^{\prime}}=\frac{R \cdot J}{R^{\prime} \cdot J}=\frac{R}{R^{\prime}} \quad \text { und } \quad \frac{A B}{A^{\prime} B^{\prime}}=\frac{r}{r^{\prime}}
$$

ist, so folgt $\frac{\mathrm{R}}{\mathrm{R}^{\prime}}=\frac{\mathrm{r}}{\mathrm{r}^{\prime}} \cdot \frac{\mathrm{I}-\operatorname{tg} \beta^{\prime} \cdot \operatorname{tg} \delta}{\mathrm{I}-\operatorname{tg} \beta \cdot \operatorname{tg} \delta}$

Für den hier wiederkehrenden Tangentenausdruck gilt das Gleiche, was bei der Ableitung der Gleichung 4a) gesagt worden ist. Wenn der Wert von $\left(\operatorname{tg} \beta-\operatorname{tg} \beta^{\prime}\right) \cdot \operatorname{tg} \delta$ kleiner als $0,00 I$ bleibt und wenn uns eine Meßgenauigkeit von $I \%$ genügt, so können wir den Ausdruck gleich I setzen, und die Gleichung I) behält ihre einfache Form

$$
R: R^{\prime}=r: r^{\prime} \text {. }
$$

Zum Schlusse sei noch auf den oft vorliegenden Sonderfall hingewiesen, daß die Strombahnen $\mathrm{AC}$ und $\mathrm{A}^{\prime} \mathrm{C}^{\prime}$ nur die Induktivitäten $\mathrm{L}$ bzw. $\mathrm{L}^{\prime}$, aber keine merkbaren Kapazitäten enthalten. Für diesen Fall läßt sich aus den Gleichungen I) und 4a) $\mathrm{L}$ in einfacher Weise auf $L^{\prime}$ zurückführen. In Gl. 4a) können wir

$$
\operatorname{tg} \beta=\frac{\omega \cdot \mathrm{L}}{\mathrm{R}} \text {. und } \quad \operatorname{tg} \beta^{\prime}=\frac{\omega \cdot \mathrm{L}^{\prime}}{\mathrm{R}^{\prime}}
$$


setzen. Sie geht dann, wenn wir den Korrektionsfaktor $I-\operatorname{tg} \beta \cdot \operatorname{tg} \delta$ zunächst beiseite lassen, in die Form über

$$
\omega \cdot \mathrm{L}=\eta \cdot \frac{\mathrm{R}}{\mathrm{R}^{\prime}} \cdot\left(\mathrm{I}+\frac{\mathrm{r}^{\prime}}{\mathrm{r}}\right) \cdot \frac{\mathrm{w}+\mathrm{w}^{\prime}}{\mathrm{w}+\mathrm{w}^{\prime}+\mathrm{W}}+\omega \cdot \mathrm{L}^{\prime} \cdot \frac{\mathrm{R}}{\mathrm{R}^{\prime}}
$$

und unter Benutzung von Gl. I):

$$
\left.\mathrm{L}=\frac{\mathrm{I}}{\omega} \cdot \eta \cdot\left(\mathrm{I}+\frac{\mathrm{r}}{\mathrm{r}^{\prime}}\right) \cdot \frac{\mathrm{w}+\mathrm{w}^{\prime}}{\mathrm{w}+\mathrm{w}^{\prime}+\mathrm{W}}+\mathrm{L}^{\prime} \cdot \frac{\mathrm{r}}{\mathrm{r}^{\prime}} \ldots . . . \quad . \quad .4 \mathrm{~b}\right)
$$

Es ist hervorzuheben, daß zur Berechnung von L nach Gl. 4 b) weder die Kenntnis von $R$ noch von $R^{\prime}$ erforderlich ist und daB die Gleichung ihre einfachste Form annimmt, wenn $L^{\prime}==\mathrm{O}$ ist. Es empfiehlt sich daher, für derartige Messungen als Vergleichswiderstand $\mathrm{A}^{\prime} \mathrm{C}^{\prime}$ einen induktions- und kapazitätsfreien Widerstand zu verwenden, der seiner Größenordnung nach in die Messanordnung paßt, dessen Wert aber nicht genau bekannt zu sein braucht. Nur zur Beurteilung der Größe von

$$
\operatorname{tg} \beta=\frac{\omega \cdot \mathrm{L}}{\mathrm{R}} \quad \text { oder } \quad=\frac{\omega \cdot \mathrm{L}}{\mathrm{R}^{\prime}} \cdot \frac{\mathrm{r}^{\prime}}{\mathrm{r}},
$$

deren Kenntnis mit Rücksicht auf die Anbringung des Korrektionsfaktors $I-\operatorname{tg} \beta \cdot \operatorname{tg} \delta$ nötig ist, muß $R$ oder $R^{\prime}$ in erster Annäherung bekannt sein.

Das Meßbereich der besprochenen Anordnung hängt neben anderem von den Größen- und Wicklungsverhältnissen des Phasenschlittens ab. In der Ausführung, wie sie in dieser Zeitschrift, Bd. V, S. 305, beschrieben worden ist, läßt sich bei 50 Perioden für $\eta$ ein Wert bis 0,25 einstellen; die Ablesung hat unterhalb des Wertes 0,05 eine Unsicherheit von mehr als I\%, bei den oberen Werten aber von nur etwa $0,2 \%$. Wählt man den Wert von $\frac{\mathrm{r}}{\mathrm{r}^{\prime}}$ zwischen $\mathrm{O}, \mathrm{I}$ und $\mathrm{I} O$, so ist bei $\mathrm{L}^{\prime}=\mathrm{O}$ die zu messende Induktivität von der Größenordnung $\mathrm{IO}^{-2}$ bis $\mathrm{IO}^{-3}$ Henry, bei $\mathrm{L}^{\prime}>\mathrm{O}$ entsprechend größer. Andere Meßbereiche werden durch andere Spulensätze des Phasenschlittens erhalten.

Will man die Gleichungen in ihrer einfachen Form, d. h. ohne Korrekturglieder, verwenden, so muß man sich auf ein $\operatorname{tg} \beta$ beschränken, das je nach der Größe von $\operatorname{tg} \delta$ und der gewünschten Meßgenauigkeit unterhalb 0,2 bis 0,4 liegt.

Zusammenfassung. Im Obigen ist gezeigt worden, wie in der Thomsonbrücke mit niederfrequentem Wechselstrom der Werkwiderstand $\mathrm{R}$ und die Phasenverschiebung a zwischen Strom und Klemmenspannung einer Strombahn, bezw. deren Induktivität L auf die entsprechenden Größen $\mathrm{R}^{\prime}, \alpha^{\prime}$ und $\mathrm{L}^{\prime}$ einer bekannten Vergleichsstrombahn zurückgeführt werden können. Dies geschieht unter Zuhilfenahme des „Phasenschlittens“ in der Schaltung der Abb. 3. Nach Einstellung auf den Galvanometerausschlag. Null gelten in erster Annäherung die Beziehungen:

nach Gl. I)

und nach Gl. 4)

$$
\mathrm{R}: \mathrm{R}^{\prime}=\mathrm{r}: \mathrm{r}^{\prime}
$$

bzw. nach Gl. 4b)

$$
\operatorname{tg} \alpha=\frac{\eta}{\mathrm{R}^{\prime}} \cdot\left(\mathrm{I}+\frac{\mathrm{r}^{\prime}}{\mathrm{r}}\right) \cdot \frac{\mathrm{w}+\mathrm{w}^{\prime}}{\mathrm{w}+\mathrm{w}^{\prime}+\mathrm{W}}+\operatorname{tg} \iota^{\prime}
$$

$$
\mathrm{L}=\frac{\mathrm{I}}{\omega} \cdot \eta \cdot\left(\mathrm{r}+\frac{\mathrm{r}}{\mathrm{r}^{\prime}}\right) \cdot \frac{\mathrm{w}+\mathrm{w}^{\prime}}{\mathrm{w}+\mathrm{w}^{\prime}+\mathrm{W}}+\mathrm{L}^{\prime} \cdot \frac{\mathrm{r}}{\mathrm{r}^{\prime}} .
$$

Je nach der Größe von $\operatorname{tg} \delta$ nach Gl. 3 und von ' $\operatorname{tg} \alpha$ und $\operatorname{tg} \alpha^{\prime}$ sind noch Korrektionsglieder anzubringen, die aus Gl. 4a) und Gl. 5) zu entnehmen sind. 